\title{
The Effects of a Constant Bias Force on the Dynamics of a Periodically Forced Spherical Particle in a Newtonian Fluid at Low Reynolds Numbers
}

\author{
K. Madhukar, "\#, T. R. Ramamohan" ${ }^{*}$ and I. S. Shivakumara ${ }^{\#}$ \\ *CSIR- Centre for Mathematical Modelling and Computer Simulation (C-MMACS), Wind Tunnel Road, \\ Bangalore - 560 037, India. \\ ${ }^{\#}$ UGC-Centre for Advanced Studies in Fluid Mechanics, Department of Mathematics, Central College, \\ Bangalore University, Bangalore - 560 001, India.
}

\begin{abstract}
We make use of the formulation developed by Lovalenti and Brady [1] for the hydrodynamic force acting upon a spherical particle undergoing arbitrary time dependent motion in an arbitrary time dependent uniform flow field at low Reynolds numbers, to derive an expression for the effects of a constant bias force acting on a periodically forced rigid spherical particle in a Newtonian fluid. We use Newton's second law to relate the total force acting on the particle to the motion of the particle. The total force is given by: Total force $=\boldsymbol{F}^{\boldsymbol{e x t}}+\boldsymbol{F}^{\boldsymbol{H}}$, where, $\boldsymbol{F}^{\boldsymbol{e x t}}$ is the external force inclusive of both the periodic force and the constant bias force. $\boldsymbol{F}^{\boldsymbol{H}}$ is the hydrodynamic force derived by Lovalenti and Brady [1] including both unsteady and convective inertia. The equation derived contains a nonlinear history term and is nonlinear. This equation is solved numerically using an adaptive step size Runge - Kutta scheme. We obtain several phase plots (plots between particle displacement and particle velocity), which show the effects of low Reynolds numbers, the periodic force and the effects of the constant bias force on the particle motion. It is observed that at low magnitudes of the periodic forcing the external constant force dominates and the particle moves along the direction of the external constant force. As we increase the magnitude of the periodic forcing, the periodic force is seen to dominate and the particle is seen to oscillate along a mean position with a slight drift along the direction of the periodic force and the external constant force, when they are imposed in the same direction. However the motion of the particle becomes more complicated when the directions of the periodic forcing and external constant force are opposite to each other. We also observe a reflection in phase space when the directions of both the forces are reversed. The phase plots typically are of a half sinusoidal, sinusoidal and a coiled (solenoidal) pattern. These plots include the effects of both periodic force and the constant bias force. As the Reynolds numbers increases the drift of the particle reduces, which indicates the effects of inertia. We present a preliminary analysis of the dynamics in this paper.
\end{abstract}

Keywords: constant bias force, periodic forcing, spherical particle and low Reynolds numbers.

PACS: 05.45.-a, 47.15.Gf, 47.11.+j

\section{INTRODUCTION}

We make use of the formulation given by Lovalenti and Brady [1] for the hydrodynamic force acting on a spherical particle undergoing arbitrary time dependent motion in an arbitrary time dependent uniform flow field at low Reynolds numbers and derive an expression for displacement and velocity of the spherical particle moving due to a constant bias force and a periodic force applied on the particle. The fluid is assumed to be quiescent and the only driving forces are the two external forces. In the next section we formulate the problem. The third section contains the methodology used to solve the integro - differential equation so obtained in the section below. We provide a validation of our results by performing appropriate tests on our software. In the next section we discuss the results obtained from our numerical simulations. We conclude by summarizing the results obtained in the last section.

\section{FORMULATION OF THE PROBLEM}

The Lovalenti and Brady [1] formalism for the hydrodynamic force on a rigid sphere undergoing arbitrary time dependent motion in an arbitrary time dependent uniform flow field at small Reynolds numbers is given by the following expression. 


$$
\begin{aligned}
& \boldsymbol{F}^{\boldsymbol{H}}(t)=\frac{4 \pi}{3} \operatorname{ReSl} \dot{\boldsymbol{U}}^{\infty}(t)-6 \pi \boldsymbol{U}_{s}(t)-\frac{2 \pi}{3} \operatorname{ReSl} \dot{\boldsymbol{U}}_{\boldsymbol{s}}(t) \\
& +\frac{3}{8}\left(\frac{\operatorname{ReS} l}{\pi}\right)^{1 / 2}\left\{\int _ { - \infty } ^ { t } \left[\frac{2}{3} \boldsymbol{F}_{\boldsymbol{s}}^{\boldsymbol{H}_{\|}}(t)-\left\{\frac{1}{|\boldsymbol{A}|^{2}}\left(\frac{\pi^{1 / 2}}{2|\boldsymbol{A}|} \operatorname{erf}(|\boldsymbol{A}|)-\exp \left(-|\boldsymbol{A}|^{2}\right)\right)\right\} \boldsymbol{F}_{\boldsymbol{s}}^{\boldsymbol{H}_{\|}}(s)\right.\right. \\
& \left.+\frac{2}{3} \boldsymbol{F}_{s}^{\boldsymbol{H}_{\perp}}(t)-\left\{\exp \left(-|\boldsymbol{A}|^{2}\right)-\frac{1}{2|\boldsymbol{A}|^{2}}\left(\frac{\pi^{1 / 2}}{2|\boldsymbol{A}|} \operatorname{erf}(|\boldsymbol{A}|)-\exp \left(-|\boldsymbol{A}|^{2}\right)\right)\right\} \boldsymbol{F}_{s}^{\boldsymbol{H}_{\perp}}\right] \\
& \left.\times \frac{2 d s}{(t-s)^{3 / 2}}\right\}+o(\operatorname{Re}) .
\end{aligned}
$$

This expression is obtained by using the reciprocal theorem. The details of the derivation and the definition of the parameters can be found in [1]. We follow the derivation given in [2] and use $\boldsymbol{U}_{\boldsymbol{p}}=\left(U_{p_{x}}, U_{p_{v}}\right)$ and Total force $=$ $F^{e x t}+F^{H}$.

We take $\boldsymbol{F}^{\text {ext }}=\left(F_{10}, F_{01}\right)+\boldsymbol{F}_{0} \sin (t)$, where $\left(F_{10}, F_{01}\right)$ is the constant bias force in arbitrary direction in the $x y$ plane. Thus substituting the expression for $\boldsymbol{F}^{\text {ext }}$ in the formulation of problem in the derivation in [2], using Newton's second law, we obtain the following equations for particle velocity and particle displacement:

$$
\begin{aligned}
& \frac{d Y_{p_{x}}}{d t}=U_{p_{x}} \\
& \frac{d Y_{p_{y}}}{d t}=U_{p_{y}} \\
& \frac{d U_{p_{x}}}{d t}=\frac{1}{\operatorname{Re}^{*}}\left[F_{10}^{*}+\operatorname{Re}_{F} \sin (t)-6 \pi U_{p_{x}}+\frac{3}{8}\left(\frac{\operatorname{Re} S l}{\pi}\right)^{1 / 2}\left(J_{1}+I_{1}\right)\right] \\
& \frac{d U_{p_{y}}}{d t}=\frac{1}{\operatorname{Re}^{*}}\left[F_{01}^{*}-6 \pi U_{p_{y}}+\frac{3}{8}\left(\frac{\operatorname{Re} S l}{\pi}\right)^{1 / 2}\left(J_{2}+I_{2}\right)\right]
\end{aligned}
$$

Here,

$$
\begin{aligned}
& J_{1}=16 \pi U_{p_{x}}(t)\left[\frac{1}{\sqrt{t}}-\frac{1}{\sqrt{\varepsilon}}\right], \\
& J_{2}=16 \pi U_{p_{y}}(t)\left[\frac{1}{\sqrt{t}}-\frac{1}{\sqrt{\varepsilon}}\right] \text { and } \\
& I_{1}=\int_{0}^{t-\varepsilon}\left\{\frac{1}{A^{2}}\left(\frac{\sqrt{\pi}}{2|A|} \operatorname{erf}(A)-\exp \left(-A^{2}\right)\right)\right\} \frac{12 \pi U_{p_{x}}(s)}{(t-s)^{3 / 2}} d s, \\
& I_{2}=\int_{0}^{t-\varepsilon}\left\{\frac{1}{A^{2}}\left(\frac{\sqrt{\pi}}{2|A|} \operatorname{erf}(A)-\exp \left(-A^{2}\right)\right)\right\} \frac{12 \pi U_{p_{y}}(s)}{(t-s)^{3 / 2}} d s .
\end{aligned}
$$

Where $\operatorname{Re}^{*}=\frac{4 \pi}{3} \operatorname{Re}+\frac{2 \pi}{3} \operatorname{Re} S l, \operatorname{Re}_{F}=\frac{F_{0}}{\mu a^{2} \omega}, F_{10}^{*}=\frac{F_{10}}{\mu a^{2} \omega}$ and $F_{01}^{*}=\frac{F_{01}}{\mu a^{2} \omega}$. 


\section{METHODOLOGY}

We developed software using Numerical Recipes in FORTRAN 77 [3] to solve equations (2.2), (2.3), (2.4) and (2.5) using an embedded Runge-Kutta method with adaptive step size. The integrals in equations (2.4) and (2.5) were evaluated at each time step by Romberg extrapolation. The function with respect to 'A' was defined by a user supplied function subprogram. We used the ODEINT, RKQS and RKCK subroutines from Numerical Recipes to implement the Runge - Kutta method. The Romberg extrapolation was performed using the QROMB subroutine. The integral was evaluated using TRAPZD and the interpolation during the numerical quadrature was performed by POLINT. The tolerance for both the Romberg extrapolation and the Runge - Kutta solver was taken as $10^{-5}$. Further reduction of the tolerance did not result in any significant change in our results. The entire program was written in double precision. The initial conditions for both the velocity and the position of the particle were taken as zero. $\varepsilon$ was taken as 0.04 ; smaller values of $\varepsilon$ did not significantly change the results. The software was tested for consistency by compiling the program with two compilers namely, Intel Fortran and F90. The results of both the programs matched except for minor differences of the order of $10^{-5}$. We generated 5000 data points taken at an interval of $\pi / 200$ in both the dimensionless velocity and dimensionless position. Further increase in the resolution did not yield any difference in our results.

In order to validate the simulation we performed all the tests given in [2] and reproduced the results of [2] by taking the constant bias force equal to zero. These tests gave us considerable confidence in our simulations.

\section{RESULTS AND DISCUSSIONS}

We have four parameters in the problem, $\mathrm{Re}, \mathrm{Re}_{\mathrm{F}}, F_{01}$ and $F_{10}$. We varied all these parameters and generated a number of displacement and velocity time series and also plots of displacement versus velocity of the particle. These plots were bounded in phase space and hence represent an attractor in the phase space. We considered 8 test cases by varying $\mathrm{Re}_{\mathrm{F}}, F_{01}$ and $F_{10}$ in the positive and negative directions and recorded the observations in Table1.

In addition to the observations in Table1, the effect of inertia on the spherical particle was evident due to the variation of Re. As Re increased, the area spanned by the phase plot decreased owing to an increase of the resistance to change in motion.
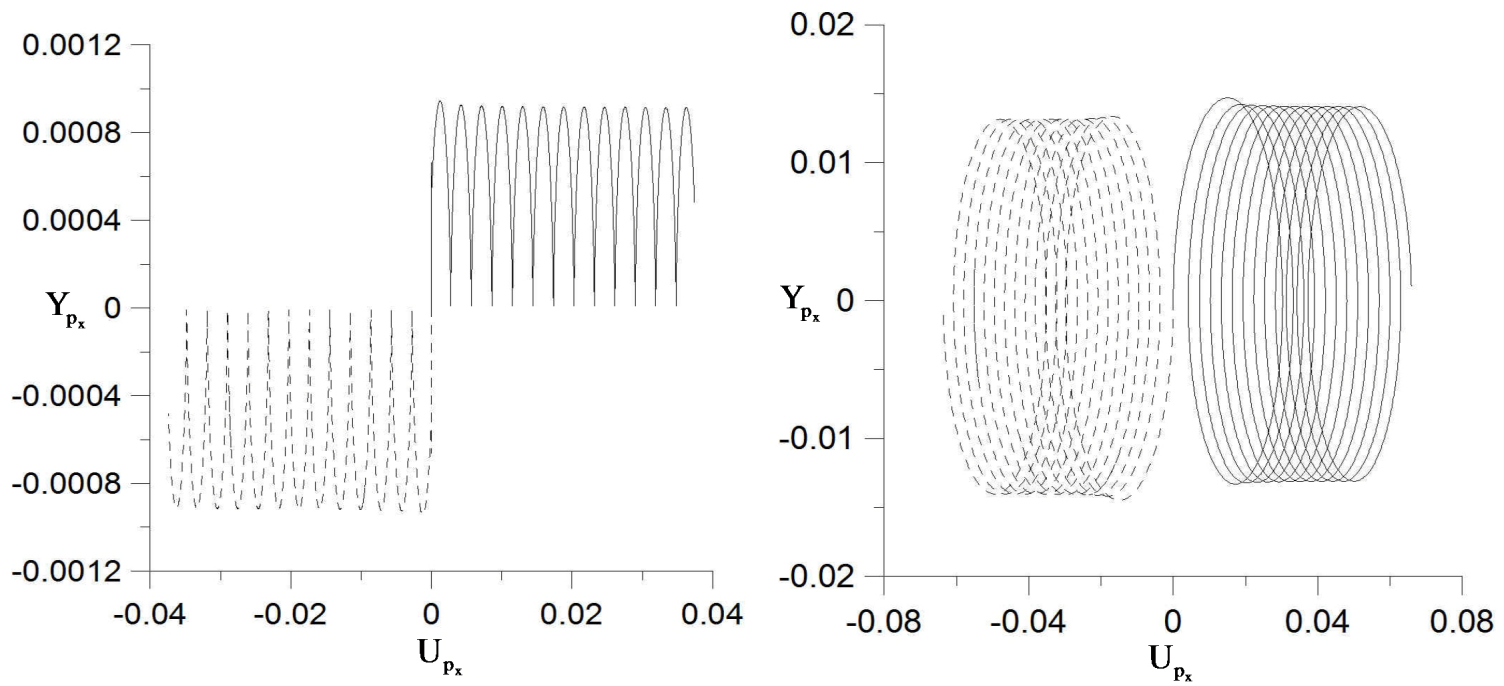

FIGURE 1. Typical phase plots for $\operatorname{Re}_{\mathrm{F}} 0.01$ and 0.3 at $F_{10}$ and $F_{01}=0.01$ and $\mathrm{Re}=0.01$. The plot also shows the reflection along the $\mathrm{y}$-axis when $\mathrm{Re}_{\mathrm{F}}$ and $F_{10}$ are taken in the negative x- direction.

\section{CONCLUSIONS}

We have obtained physically valid results from our simulations. We observed that increase in Re increases the resistance to change in motion and hence lowering the motion of the particle. Increase in $\mathrm{Re}_{\mathrm{F}}$, increases the oscillations in the motion of the spherical particle and at high $\mathrm{Re}$ and high $\operatorname{Re}_{\mathrm{F}}$ the particle oscillates along a mean position. It is obvious that when the constant bias force is along the periodic force then there is a drift of the 
spherical particle along the same direction. If $\mathrm{Re}_{\mathrm{F}}$ is greater than $F_{10}$, then the phase plots are solenoidal (coiled), which means oscillations with fewer drift. If $F_{10}$ is larger than the phase plot is sinusoidal with less amplitude. When $F_{10}$ is equal to $\mathrm{Re}_{\mathrm{F}}$ then we get half sinusoidal waves owing to a large drift of the spherical particle. If $F_{10}$ and $\operatorname{Re}_{\mathrm{F}}$ are in the opposite directions then we observe the same pattern in the $2^{\text {nd }}$ and $3^{\text {rd }}$ quadrant of the $x y-$ plane. Since periodic forcing is not present along the $\mathrm{y}$-axis, the effect of $F_{01}$ is predominantly linear and constant.

TABLE 1. Behavior of the spherical particle due to variations in sign of $\operatorname{Re}_{\mathrm{F}}, F_{10}, F_{01}$. (+ represents positive; - represents negative directions)

$$
\operatorname{Re}_{\mathrm{F}} \quad F_{10} \quad F_{01} \quad \text { Motion of the spherical particle }
$$

\footnotetext{
At low $\mathrm{Re}_{\mathrm{F}}$, a drift of the particle along the direction of $F_{10}$ is observed. The phase plots show sinusoidal and $+\mathrm{x}+\mathrm{x} \quad+\mathrm{y}$ half sinusoidal waves. At high $F_{01}$, the motion becomes linear along the $\mathrm{x}-\mathrm{axis}$. At high Re $\mathrm{F}_{\mathrm{F}}$, a coiled phase plot is observed, which shows that the particle is oscillating and drifting in the direction of $F_{10}$.

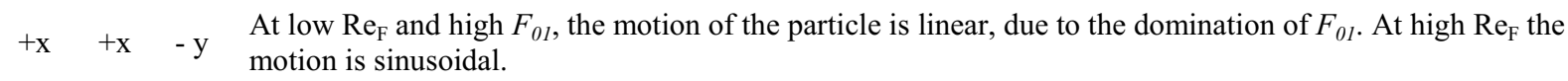

$+\mathrm{x} \quad-\mathrm{x} \quad+\mathrm{y}$ axis. At high $F_{10}$ a half sinusoidal pattern observed along $-\mathrm{x}-$ axis. A mirror image of $+\mathrm{x}$ values of $F_{10}$ is observed in the $3^{\text {rd }}$ quadrant.
Since both $F_{10}$ and $F_{01}$ are negative, the x- component of $\mathrm{U}_{\mathrm{p}}$ shows oscillations for high $\mathrm{Re}_{\mathrm{F}}$ and sinusoidal $+\mathrm{x}-\mathrm{x}-\mathrm{y}$ pattern for low $\mathrm{Re}_{\mathrm{F}}$ along the $-\mathrm{x}$ - axis. The $\mathrm{y}-$ component of $\mathrm{U}_{\mathrm{p}}$ shows a mirror image of the $\mathrm{y}-$ component of $\mathrm{U}_{\mathrm{p}}$ with $+\mathrm{x} F_{10}$ and $+\mathrm{y} F_{01}$.

$-\mathrm{x}+\mathrm{x}+\mathrm{y}$ High $\operatorname{Re}_{\mathrm{F}}$, oscillations from $+\mathrm{x}$ to $-\mathrm{x}$ axis with mean zero. $F_{10}$ forces the particle along $+\mathrm{x}$ and $\mathrm{Re}_{\mathrm{F}}$ along $-\mathrm{x}$, hence the mean is nearly zero. At low $\mathrm{Re}_{\mathrm{F}}$, phase plot shows a half sine pattern with mean zero.

$-\mathrm{x}+\mathrm{x}-\mathrm{y}$ The $\mathrm{y}-$ component of $\mathrm{U}_{\mathrm{p}}$ gives the exact reflection of the $\mathrm{y}$ - component of $\mathrm{U}_{\mathrm{p}}$ with $+\mathrm{x} \operatorname{Re}_{\mathrm{F}}$ and $+\mathrm{y} F_{01}$. The $\mathrm{x}$ component shows no change from above result, i.e., $-\mathrm{x} \mathrm{Re}_{\mathrm{F}}$ and $+\mathrm{x} F_{10}$.

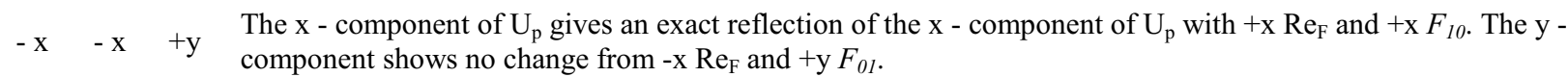

$-\mathrm{x} \quad-\mathrm{x} \quad-\mathrm{y} \quad$ Reflection of $+\mathrm{x} \mathrm{Re}_{\mathrm{F}},+\mathrm{x} F_{10}$ and $+\mathrm{y} F_{01}$. i.e., motion of the particle along $-\mathrm{x}$ and $-\mathrm{y}$ axis.
}

\section{ACKNOWLEDGMENTS}

The authors wish to acknowledge Dr. A R Upadhya, Scientist - in - Charge, CSIR-CMMACS, Bangalore-560037, India and Prof. N. Rudraiah, Honorary Professor, UGC - CAS, Department of Mathematics, Bangalore University, Bangalore - 560001, India, for their kind encouragement. One of the authors, K. Madhukar wishes to thank NAL/CMMACS for providing the fellowship to do this research work.

\section{REFERENCES}

1. P M Lovalenti and J F Brady, J. Fluid Mech., 256, 561 - 605 (1993).

2. T R Ramamohan, I S Shivakumara and K Madhukar, LNCS 5544, 591 - 600 (2009).

3. W H Press, S A Teukolsky, W T Vetterling, B P Flannery. Numerical recipes in FORTRAN 77, second edition. The art of scientific computing. Cambridge University Press. (1992). 